\title{
In Situ Fiber-Optic Dissolution Assisted by a Mathematical Separation Model of Dynamic Three-Wavelength K-Ratio Spectrophotometry
}

\author{
Kun Nie ${ }^{1,2}$, Li Li ${ }^{1}, *$, Xinxia Li ${ }^{1}$, Dongsheng Geng ${ }^{3}$, Qizhou Zhang ${ }^{4}$, \\ Mingfu Tuo ${ }^{1}$, Pingping $\mathrm{Xu}^{1}{ }^{1}$, and Jian $\mathrm{Chen}^{1}$ \\ ${ }^{1}$ School of Pharmacy, Xinjiang Medical University, Urumqi, Xinjiang 830011 , China \\ 2 Jinan Cinit Science and Technology Co., Ltd, Jinan, Shandong 250101, China \\ ${ }^{3}$ Institute for Drug and Instrument Control, United Logistics Department of Xinjiang Command, PLA, Urumqi, Xinjiang 830063, China \\ ${ }^{4}$ Xinjiang FOCS Bio-tech Development Co., Ltd., Urumqi, Xinjiang 830031, China
}

\begin{abstract}
The aim of this study was to monitor the drug release of ibuprofen sustained-release capsules in real time in situ. A mathematical separation model of dynamic three-wavelength $K$-ratio spectrophotometry was established to eliminate the interference of gelatin capsule shells when drug release was monitored by a fiber-optic drug dissolution in situ test system. A control experiment with high performance liquid chromatography was performed simultaneously. Within $7 \mathrm{~h}$, real-time drug release profiles were obtained. The average drug release rates were $19.79 \%, 37.34 \%, 63.13 \%$, and $88.41 \%$ by the fiber-optic drug dissolution in situ test system at the respective time points of $1 \mathrm{~h}, 2 \mathrm{~h}, 4 \mathrm{~h}$, and $7 \mathrm{~h}$. There were no significant differences between the in situ and control methods. Drug release of ibuprofen sustained-release capsules can be monitored by the fiber-optic drug dissolution in situ test system assisted by the mathematical separation model of dynamic three-wavelength $K$-ratio spectrophotometry. Information about the entire dissolution process can be produced by the real-time release profile.
\end{abstract}

\section{INTRODUCTION}

he determination of the release rate of active
ingredients from drug formulations is an important
procedure in pharmaceutical research. Without
automation, it is a labor-intensive process. The fiber-optic
sensor based on ultraviolet-visible (UV-vis) absorption
analysis for dissolution testing is a technique that has
been under investigation for many years (1-3). Drug
dissolution process analysis with fiber-optics uses a
fiber-optic probe inserted directly into each vessel, and
the absorbance of the solution is measured in situ instead
of extracting samples and determining the concentration
of active ingredient with UV spectrophotometry or high
performance liquid chromatography (HPLC). This
approach is rapid and efficient, with less handling of the
samples and consequently fewer sources of error.
A pharmaceutical tablet, however, always contains a
number of other compounds (i.e., excipients), and
sometimes these compounds can influence the
determination of the active ingredient. When samples
from the dissolution test are analyzed without any
separation, excipients that have absorbance at the same
wavelength as the drug may have a large influence on the
dissolution data. Scattering can be generated from
undissolved particles and excipients, which can
contribute significant interference. This type of
scattering may be either wavelength-independent

*Corresponding author. or wavelength-dependent. Both of the two interferences can be eliminated by some algorithm (4). The use of fiber optics, an automatic controlling system, and software, together with powerful chemometric data evaluation, can open up new possibilities for a deeper understanding of in vitro dissolution testing (5).

In 1988 a partial-least-squares (PLS) statistical method was used to correct for the disturbance caused by solution turbidity in dissolution testing (1). In 2000 a fiber-optic sensor system was utilized by Chen et al. (6) for on-line dissolution monitoring of multicomponent solid preparations containing vitamins $B_{1}, B_{2}$, and $B_{6}$. In addition, in 2008 it was reported that thirty-five excipient samples and five placebos were selected to evaluate the Delphian fiber-optic dissolution system in the wavelength range of 200-400 nm (3).

Fenbid (Ibuprofen Sustained-Release Capsules), a product of GlaxoSmithKline (Sino-American Tianjin SmithKline and French Lab., Ltd), is an ibuprofen pain reliever featuring a 12 -h sustained-release formula. The absorbance of the gelatin capsule shells at $264 \mathrm{~nm}$ can interfere with the measurements of ibuprofen, which has a maximum absorbance at about $264 \mathrm{~nm}$. The vessels were sampled at $1,2,4$, and $7 \mathrm{~h}$, then determined by HPLC according to the Chinese Pharmacopeia 2005 (Ch.P) (7). In this paper, a mathematical separation model of dynamic three-wavelength $F$-ratio spectrophotometry, which can eliminate the interference of gelatin capsule shells, was investigated. The drug release of ibuprofen 
sustained-release capsules can be monitored in real time in situ with a fiber-optic drug dissolution test system. The in situ dissolution data were compared with data determined by a control off-line HPLC assay. There were no significant differences between the in situ and control methods.

\section{MATERIALS AND METHODS Instruments}

A fiber-optic drug dissolution test system (FODT) was developed by Chen et al. (6) of Xinjiang Medical University and Xinjiang FOCS Biotech Development Co., Ltd. The system was strictly validated for both the UV-vis spectrophotometry and dissolution test methods of the Ch.P.It consists of a deuterium $\left(D_{2}\right)$ lamp, six fiber-optic dipping probes, and a detector with a charge-coupled device (CCD) that can collect data at each wavelength point. Light from the deuterium lamp passes through incident optical fibers, which transfer the light from the source to the probes immersed in the vessels during testing. Light again reflects back from the mirror at the bottom of the probe tip to the CCD through the detection fiber. The probe path can be altered to $0.5,1.0$, 2.0, and $5.0 \mathrm{~mm}$ to adjust the sensitivity. The UV-vis spectra (220-600 nm) from six fiber channels with low noise can simultaneously be collected with the system. Data acquisition and processing was accomplished by the software, which was compiled by Xinjiang FOCS Biotech Development Co., Ltd.

A spectrophotometer (Cintra 40, GBC, Australia) was utilized for the spectral scans, and an HPLC (LC-20AT, Shimadzu, Japan) for the off-line control determination.

\section{Reagents and Drugs}

The purified water was made in the laboratory and degassed before use. Sodium hydroxide, potassium dihydrogen phosphate, and hydrochloric acid were all analytical reagents obtained from Fuchen Chemical Reagents (Tianjin, China). Ibuprofen reference substance (Batch No.100179-200303) was from the National Institute for the Control of Pharmaceutical and Biological Products of China.

Ibuprofen sustained-release capsules (Fenbid, SinoAmerican Tianjin SmithKline and French Lab., Ltd, 300 mg, Batch No. 08040807) were from the commercial market.

\section{UV Spectral Characteristics of Ibuprofen}

Solutions of ibuprofen reference substance, gelatin capsule shells, and ibuprofen sustained-release capsules were prepared with phosphate buffer $(\mathrm{pH} 6.0)$ to be scanned by UV spectrophotometry.

The determination data of ibuprofen reference substance, gelatin capsule shells, and ibuprofen sustainedrelease capsules were obtained from UV spectra, which is the foundation for establishing the mathematical separation model of dynamic three-wavelength $K$-ratio spectrophotometry.

\section{Linearity}

A channel was chosen to study linearity with a series of different concentrations of ibuprofen substance at $19.23 \%$, $38.46 \%, 57.69 \%, 76.92 \%, 96.16 \%$, and $115.39 \%$ according to the established method.

\section{Precision and Recovery}

Solutions at high, middle, and low concentrations were determined five times in one day continuously and once daily for five days to calculate the precision.

Samples at high, middle, and low concentrations with backgrounds that were withdrawn from the release solution were determined to calculate the recovery.

\section{Drug Release Test Methods}

Drug release testing was performed in $900 \mathrm{~mL}$ of phosphate buffer ( $\mathrm{pH}$ 6.0) at $37 \pm 0.5^{\circ} \mathrm{C}$ for $7 \mathrm{~h}$ with Ch.P Apparatus I, the same as USP Apparatus 1.The stirring speed was $30 \mathrm{rpm}$. The sampling interval for the FODT was preset at $5 \mathrm{~min}$. The real-time drug release data and profiles were acquired within $7 \mathrm{~h}$.

For HPLC analysis, the sampling time points were set at $1,2,4$, and $7 \mathrm{~h}$. Samples were extracted from the dissolution vessels and filtered through $0.45-\mu \mathrm{m}$ filters, and then injected into the HPLC.

\section{RESULTS}

\section{UV Spectral Characteristics}

Figure 1 shows UV spectra of ibuprofen reference substance, gelatin capsule shells, and ibuprofen sustained-release capsules with a concentration of $100 \%$ of the labeled amount for the formulated product, equivalent to dissolving one capsule in $900 \mathrm{~mL}$ of phosphate buffer ( $\mathrm{pH}$ 6.0). Probes with a path of $5 \mathrm{~mm}$ were utilized according to the absorbance at the maximum absorption wavelength. Ibuprofen has an absorbance maximum at $264 \mathrm{~nm}$. UV spectra revealed that the gelatin capsule shell has absorbance maxima at 264 and $425 \mathrm{~nm}$, and strong-end absorbance below $250 \mathrm{~nm}$, while the other excipients do not interfere with the measurements after baseline correction.

According to the HPLC analysis method in Ch.P, samples were determined at $263 \pm 1 \mathrm{~nm}$ after chromatographic separation; however, the absorbance of the gelatin capsule shell interferes with the ibuprofen measurement when the determination was performed with FODT. In order to monitor the release in situ without any physical or chemical separation, a mathematic method should be utilized.

Our FODT system utilizes a CCD detector that can simultaneously determine absorbance at multiple wavelengths. The absorbance of gelatin capsule shells at both $264 \mathrm{~nm}$ and $425 \mathrm{~nm}$ was determined, and a $K$-value was defined whereby $A_{264}=K \cdot A_{425}$. The $K$-value for the gelatin capsule shell was determined with the same dissolution condition as for ibuprofen sustained-release capsules before a release test. 


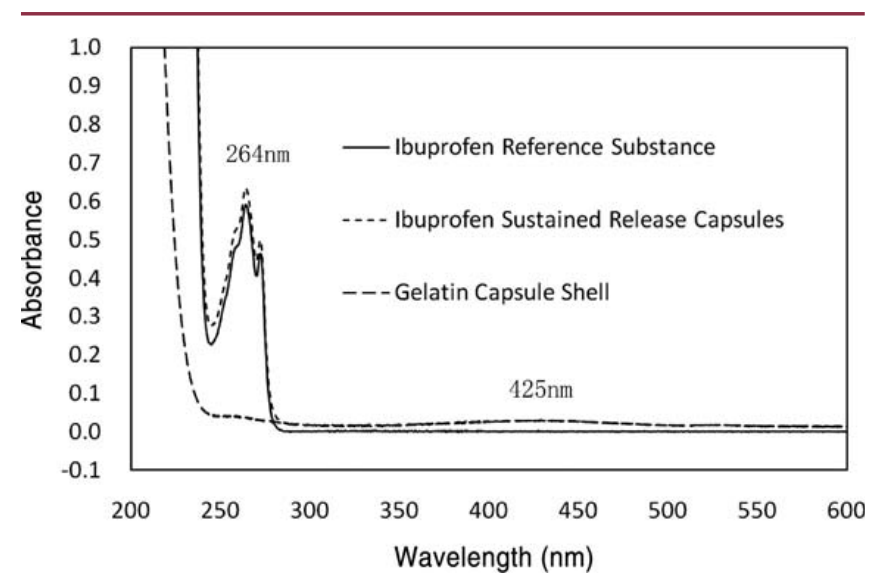

Figure 1.UV absorbance spectra of ibuprofen reference substance, gelatin capsule shell, and ibuprofen sustained-release capsules.

In the following equations, I refers to ibuprofen from ibuprofen sustained-release capsules and // refers to the gelatin capsule shell. The absorbance of ibuprofen I $(A)$ was calculated using the equations

$$
\begin{aligned}
& A=A_{1}-K A_{2}=\left(A_{264}-A_{550}\right)-K\left(A_{425}-A_{550}\right) \\
& =A_{264}^{\prime}+A_{264}^{\prime \prime}-A_{550}-K\left(A_{425}^{\prime \prime}-A_{550}\right) \quad\left(\because A_{264}^{\prime \prime}=K A_{425}^{\prime \prime}\right) \\
& =A_{264}^{\prime}+(K-1) A_{550}
\end{aligned}
$$

where $A_{1}$ and $A_{2}$ are the absorbances determined at $264 \mathrm{~nm}$ and $425 \mathrm{~nm}$, and $A_{550}$ is the background absorbance signal. $A_{550}$ was subtracted to correct small baseline offsets due to the turbid system. The correction wavelength could be set at $550 \mathrm{~nm}$ or any other at which there is no absorbance. Therefore, the absorbance calculated in the vessels depends only on the absorbance of ibuprofen active ingredient at $264 \mathrm{~nm}$ and the baseline offset. A mathematical separation model of dynamic K-ratio three-wavelength spectrophotometry was constructed to eliminate the interference of gelatin

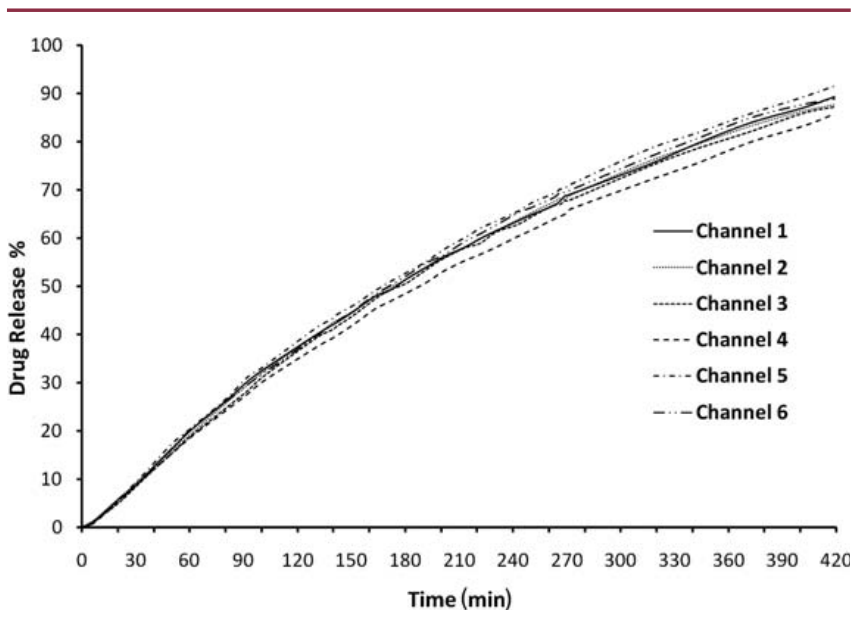

Figure 2. Release profiles of ibuprofen sustained-release capsules over $7 \mathrm{~h}$.
Table 1. Comparison of FODT and HPLC Methods for Ibuprofen Sustained-Release Tablets Release $(n=6)$

\begin{tabular}{lccccc}
\hline & \multicolumn{2}{c}{ FODT } & & \multicolumn{2}{c}{ HPLC } \\
\cline { 2 - 3 } \cline { 5 - 6 } Time (h) & Release (\%) & RSD (\%) & & Release (\%) & RSD (\%) \\
\hline 1 & 19.79 & 3.66 & & 18.68 & 2.46 \\
\hline 2 & 37.34 & 3.28 & & 36.12 & 3.34 \\
\hline 4 & 63.13 & 2.48 & & 61.75 & 2.36 \\
\hline 7 & 88.41 & 2.24 & & 87.42 & 2.51 \\
\hline
\end{tabular}

capsule shells in monitoring drug release of ibuprofen sustained-release capsules in real time in situ by FODT.

\section{Linearity}

After calculation, a regression equation was performed, $C(\%)=0.2+162.8$ A. The correlation coefficient $(r)$ was 0.99996 for concentrations from $19.23 \%$ to $115.39 \%$.

\section{Precision and Recovery}

The RSDs of intra- and interday determinations were less than $1.14 \%$. The recovery values ranged from $98.97 \%$ to $102.07 \%$, and the average RSD was $0.92 \%$. The results demonstrate that the determination was unaffected by the gelatin capsule shell.

\section{Drug Release Results}

Figure 2 shows the real-time release profiles of ibuprofen sustained-release capsules over 7 h. Average release data of six channels of ibuprofen sustained-release tablets obtained by FODT and the control method (HPLC) are shown in Table 1. There was no significant difference between the FODT and the HPLC methods $(P>0.05)$.

\section{DISSCUSION AND CONCLUSION}

A fiber-optic drug dissolution in situ test system was proved useful for pharmaceutical solid formulations. Drug dissolution-release profiles were obtained in situ by FODT and displayed in real time on the computer screen. With the mathematical separation model of dynamic three-wavelength $K$-ratio spectrophotometry, dissolution testing of ibuprofen sustained-release capsules produced results that were in good agreement with HPLC.

After the release testing of gelatin capsule shells, the constant $K$ was obtained. Therefore, $K$ calculations were set in the program, and a mathematical separation model of dynamic three-wavelength $K$-ratio spectrophotometry, which can dynamically monitor the drug release of ibuprofen in situ by FODT, was constructed.

This mathematical separation model can also be utilized to eliminate interference from other ingredients or be expanded to apply to a multicomponent product. Drug dissolution of multicomponents with several partially overlapping but different spectra can also be monitored using other algorithms for multivariate calibration, thus 
the need for separation with HPLC will be replaced in some dissolution tests.

It was reported that a second-derivative pretreatment was utilized to eliminate the problem of shifting baselines when ibuprofen release from multiparticulates was monitored by the in situ fiber-optic technique (8). However, because of limitations in instrument capability and accuracy, the wavelength and absorbance values have some fluctuations that can enlarge the errors in derivative spectrometry, especially at higher orders. The $K$-ratio method that we report here for eliminating the excipient disturbance does not perform a derivative transformation and is not affected by derivative-related errors.

Although UV determination is a common method for dissolution analysis, it involves sample removal and manipulation, which can be potential sources of error. For example, the active component might be adsorbed onto the sample filter causing an error in the amount of dissolved drug. The HPLC method, which is commonly employed for dissolution analysis, also has shortcomings (e.g., is labor-intensive and time-consuming). This FODT system has high potential application for sustained-release preparations because of its ability to perform with great stability over many hours or even several days. However, an FODT-based method cannot replace HPLC completely for drugs that have no UV-vis absorbance.

\section{ACKNOWLEDGMENTS}

Authors would like to acknowledge Xinjiang FOCS

Biotech Co., Ltd., China, for its contribution to this work.

This work was supported by National Key Project of

Scientific and Technical Supporting Programs Funded by Ministry of Science \& Technology of China during the 11 th
Five-year Plan (NO.2006BAK03A16) and National Natural Science Foundation of China (No.30960365).

\section{REFERENCES}

1. Josefson, M.; Johansson, E.; Torstensson, A. Optical fiber spectrometry in turbid solutions by mutltivariate calibration applied to tablet dissolution testing. Anal. Chem. 1988, 60 (24), 2666-2671.

2. Cho, J.H.; Gemperline, P. J.; Salt, A.;Walker, D. S. UV/ Visible Spectral Dissolution Monitoring by In Situ Fiber-Optic Probes. Anal. Chem. 1995, 67 (17), 2858-2863

3. Liu, L.; Fitzgerald, G.; Embry, M.; Cantu, R.; Pack, B. Technical evaluation of a fiber-optic probe dissolution system. Dissolution Technol. 2008, 15 (1), 10-20.

4. Lu, X.; Lozano, R.; Shah, P. In-situ dissolution testing using different UV fiber optic probes and instruments. Dissolution Technol. 2003, 10 (4), 6-15.

5. Wiberg, K.H.; Hultin, U.K. Multivariate chemometric approach to fiber-optic dissolution testing. Anal. Chem. 2006, 78 (14), 5076-5085.

6. Li, W.; Chen, J.; Xiang, B.; An, D. Simultaneous on-line dissolution monitoring of multicomponent solid preparations containing vitamins $\mathrm{B} 1, \mathrm{~B} 2$, and $\mathrm{B} 6$ by a fiber-optic sensor system. Anal. Chim. Acta 2000, 408, 39-47.

7. Pharmacopeia Committee of the Ministry of Public Health. The Chinese Pharmacopeia; Chemical Industry Press: Beijing, 2005.

8. Bijlani, V.; Yuonayel, D.; Katpally, S.; Chukwumezie, B. N.; Adeyeye, M.C. Monitoring ibuprofen release from multiparticulates: in situ fiber-optic technique versus the HPLC method: a technical note. AAPS PharmSciTech 2007, 8 (3), E9-E12. 